\title{
OPTIMAL LOGARITHMIC ENERGY POINTS ON THE UNIT SPHERE
}

\author{
J. S. BRAUCHART
}

\begin{abstract}
We study minimum energy point charges on the unit sphere $\mathbb{S}^{d}$ in $\mathbb{R}^{d+1}, d \geq 3$, that interact according to the logarithmic potential $\log (1 / r)$, where $r$ is the Euclidean distance between points. Such optimal $N$-point configurations are uniformly distributed as $N \rightarrow \infty$. We quantify this result by estimating the spherical cap discrepancy of optimal energy configurations. The estimate is of order $\mathcal{O}\left(N^{-1 /(d+2)}\right)$. Essential is an improvement of the lower bound of the optimal logarithmic energy which yields the second term $(1 / d)(\log N) / N$ in the asymptotical expansion of the optimal energy. Previously, this was known for the unit sphere in $\mathbb{R}^{3}$ only. Furthermore, we present an upper bound for the error of integration for an equallyweighted numerical integration rule $\mathrm{Q}_{N}$ with the $N$ nodes forming an optimal logarithmic energy configuration. For polynomials $p$ of degree at most $n$ this bound is $C_{d}\left(N^{1 / d} / n\right)^{-d / 2}\|p\|_{\infty}$ as $n / N^{1 / d} \rightarrow 0$. For continuous functions $f$ of $\mathbb{S}^{d}$ satisfying a Lipschitz condition with constant $C_{f}$ the bound is $\left(12 d C_{f}+C_{d}^{\prime}\|f\|_{\infty}\right) \mathcal{O}\left(N^{-1 /(d+2)}\right)$ as $N \rightarrow \infty$.
\end{abstract}

\section{INTRODUCTION AND STATEMENT OF RESULTS}

In this paper we want to study the distribution of points $\mathbf{x}_{1}, \ldots, \mathbf{x}_{N}$ on the unit sphere $\mathbb{S}^{d}$ in $\mathbb{R}^{d+1}, d \geq 2$, that maximizes the product of all mutual pairwise Euclidean distances

$$
\prod_{j \neq k}\left|\mathbf{x}_{j}-\mathbf{x}_{k}\right|
$$

or equivalently, minimizes the logarithmic energy

$$
\mathrm{E}\left(X_{N}\right):=\sum_{j \neq k} \log \frac{1}{\left|\mathbf{x}_{j}-\mathbf{x}_{k}\right|}
$$

over all $N$-point sets on $\mathbb{S}^{d}$ as $N$ goes to infinity. For the same problem on the unit sphere in $\mathbb{R}^{3}$ we refer to the literature (for example 7, 8, 26, 30, 31). The fast generation of nearly minimum logarithmic energy configurations is Problem 7 of Smale's "mathematical problems for the next century" [29.

Received by the editor April 19, 2007 and, in revised form, June 17, 2007.

2000 Mathematics Subject Classification. Primary 41A25; Secondary 31B15, 33C45, 70F10.

Key words and phrases. Discrepancy, Fekete points, logarithmic energy, Riesz energy, sphere, ultraspherical expansion.

The research of this author was supported, in part, by the U. S. National Science Foundation under grant DMS-0532154 (D.P. Hardin and E.B. Saff principal investigators).

(C)2008 American Mathematical Society Reverts to public domain 28 years from publication 
The discrete logarithmic energy of an $N$-point set is a limit case (as $s \rightarrow 0$ ) of the Riesz s-energy

$$
\mathrm{E}_{s}\left(X_{N}\right):=\sum_{j \neq k} \frac{1}{\left|\mathbf{x}_{j}-\mathbf{x}_{k}\right|^{s}}
$$

by means of $\left(1 /|\mathbf{x}|^{s}-1\right) / s \rightarrow \log (1 /|\mathbf{x}|)$ as $s \rightarrow 0$. The Riesz $s$-energy of point sets will play a role in our proofs. For more details we recommend the excellent survey articles [11, 27].

From classical potential theory [19] it follows that optimal logarithmic energy points are (asymptotically) uniformly distributed over $\mathbb{S}^{d}$ as $N$ goes to infinity. Points are uniformly distributed in the sense that any reasonable subset of $\mathbb{S}^{d}$ gets a fair share of points. We make this more precise below. In this paper we include a proof of the well-distribution property using a non-potential theoretical argument based on discrepancy of point sets. Essentially, the discrepancy of a point set measures the quality of the discrete point distribution with respect to a family of test sets (for example spherical caps). Spherical cap discrepancy tending to zero is one of several equivalent characterizations of uniform distribution summarized in Lemma 1.4. We quantify uniform distribution of optimal logarithmic points by estimating their spherical cap discrepancy which is of order $\mathcal{O}\left(N^{-1 /(d+2)}\right)$ as $N \rightarrow \infty$ (Theorem 1.6). The discrepancy is bounded by using improved lower bounds of the $N$-point minimum logarithmic energy of $\mathbb{S}^{d}$

$$
\mathcal{E}(N):=\inf \left\{\mathrm{E}\left(X_{N}\right) \mid X_{N}=\left\{\mathbf{x}_{1}, \ldots, \mathbf{x}_{N}\right\} \subseteq \mathbb{S}^{d}\right\} .
$$

These lower bounds lead to the correct second order term $(1 / d)(\log N) / N$ of the asymptotical expansion of $\mathcal{E}(N)$ for large $N$ (Lemma 1.1. Theorem 1.2). Previously, this was known for $\mathbb{S}^{2}$ in $\mathbb{R}^{3}$.

First we establish an estimate of the $N$-point minimum logarithmic energy of $\mathbb{S}^{d}$. The right-hand side of (1.2) can be seen as the discrete energy of the counting measure $\mu_{N}$ which places the point mass $1 / N$ at each point $\mathbf{x}_{j}, j=1, \ldots, N$. Similarly, the logarithmic energy of a (Radon) probability measure $\mu$ supported on $\mathbb{S}^{d}$ is given by

$$
\mathcal{I}[\mu]:=\iint \log \frac{1}{|\mathbf{x}-\mathbf{y}|} \mathrm{d} \mu(\mathbf{x}) \mathrm{d} \mu(\mathbf{y}) .
$$

By classical potential theory, the energy $\mathcal{I}[\mu]$ is uniquely minimized by the normalized surface area measure $\sigma, \int_{\mathbb{S}^{d}} \mathrm{~d} \sigma=1$. The $N$-point minimum logarithmic energy of $\mathbb{S}^{d}$ satisfies

$$
\mathcal{I}[\sigma]-\frac{1}{2} \frac{\log N}{N}+\frac{c_{1}}{N} \leq \frac{\mathcal{E}(N)}{N^{2}} \leq \mathcal{I}[\sigma]-\frac{1}{d} \frac{\log N}{N}+\frac{c_{2}}{N}
$$

with some constants $c_{1}, c_{2}$ depending on $d$ only. The lower bound follows from 32. The upper bound follows from an averaging argument 17] based on equal area partitions [20]. These bounds give the correct form of the second order term in the asymptotics of the $N$-point minimum logarithmic energy of $\mathbb{S}^{2}$. In this paper we show:

Lemma 1.1. Let $d \geq 2$. Then

$$
\frac{\mathcal{E}(N)}{N^{2}} \geq \mathcal{I}[\sigma]-\frac{1}{d} \frac{\log N}{N}-\frac{C_{d}^{\prime}}{N}+\mathcal{O}_{\varepsilon}\left(N^{-1-2 \varepsilon / d}\right), \quad N \rightarrow \infty,
$$

where $C_{d}^{\prime}>0$, given by (2.18), does not depend on $N$. 
The number $\varepsilon$ satisfies $0<\varepsilon<1$ ( $d$ even) or $0<\varepsilon<1 / 2$ ( $d$ odd). This result follows from Lemma 2.1. Combining the upper bound in (1.5) and Lemma 1.1 we obtain the following asymptotical expansion of the $N$-point minimum logarithmic energy of $\mathbb{S}^{d}$.

Theorem 1.2. Let $d \geq 2$. Then

$$
\frac{\mathcal{E}(N)}{N^{2}}=\mathcal{I}[\sigma]-\frac{1}{d} \frac{\log N}{N}+\mathcal{O}\left(N^{-1}\right), \quad N \rightarrow \infty .
$$

Remark 1.3. The proof of Theorem 1.2 shows that the geometrical structure of optimal logarithmic energy points does not play a role in the derivation of the second term of the asymptotics of $\mathcal{E}(N)$. For the $N$-point minimal Riesz $s$-energy $\mathcal{E}_{s}(N), 0<s<d$, similar ideas used here produce the negative sign and the correct order $N^{1+s / d}$ for the second term (cf. [4]). It is still not clear whether the second term of the asymptotics exists for $0<s<d$ or not. For $\mathbb{S}^{2}$ it is conjectured in 17. that the second term for $\mathcal{E}_{s}(N)$ is given by $-[\sqrt{3} /(8 \pi)]^{s / 2} \zeta_{\Lambda}(s) N^{1+s / d}$ and $\zeta_{\Lambda}$ denotes the zeta function associated with the hexagonal lattice.

Next, we discuss the concept of uniform distribution on the unit sphere in $\mathbb{R}^{d+1}$ which is closely related to numerical integration on the $d$-sphere. The exact integral of a (continuous) function defined on $\mathbb{S}^{d}$ is denoted by

$$
\mathrm{I}[f]:=\int_{\mathbb{S}^{d}} f(\mathbf{x}) \mathrm{d} \sigma(\mathbf{x})
$$

where $\sigma$ is the surface area measure on the $d$-sphere normalized such that $\int_{\mathbb{S}^{d}} \sigma=$ 1. The exact integral $\mathrm{I}[f]$ can be approximated by equally-weighted numerical integration formulas $Q_{N}$ with nodes $\mathbf{x}_{j} \in \mathbb{S}^{d}$,

$$
\mathrm{Q}_{N}[f]:=\frac{1}{N} \sum_{j=1}^{N} f\left(\mathbf{x}_{j}\right)
$$

The members of a sequence $X_{N}, N \geq 2$, of $N$-point configurations on the sphere $\mathbb{S}^{d}$ are said to be (asymptotically) uniformly distributed if

$$
\lim _{N \rightarrow \infty} \mathrm{Q}_{N}[f]=\mathrm{I}[f]
$$

for all functions $f$ continuous on the sphere. An equivalent characterization is that for every $\sigma$-measurable set $B \subseteq \mathbb{S}^{d}$ with $\mathcal{H}^{d}(\partial B)=0$ there holds that

$$
\frac{\left|X_{N} \cap B\right|}{N} \rightarrow \sigma(B), \quad N \rightarrow \infty
$$

That means, as $N$ becomes large, any such $B$ gets a fair share of points. $\mathcal{H}^{d}(\cdot)$ denotes the $d$-dimensional Hausdorff measure.

Relation (1.6) yields a natural measure for the quality of the distribution of an $N$-point set $X_{N}$. The $\mathcal{F}$-discrepancy

$$
D\left(\mathcal{F} ; X_{N}\right):=\sup _{B \in \mathcal{F}}\left|\frac{\left|X_{N} \cap B\right|}{N}-\sigma(B)\right|
$$

gives the maximal deviation between the discrete point distribution and the uniform $\sigma$ with respect to a reasonable family $\mathcal{F}$ of test sets $B \subseteq \mathbb{S}^{d}$. If the test sets are spherical caps with center $\mathbf{x}$ and opening $-1 \leq t \leq 1$,

$$
C_{t}(\mathbf{x}):=\left\{\mathbf{y} \in \mathbb{S}^{d} \mid\langle\mathbf{x}, \mathbf{y}\rangle \geq 1\right\}
$$

we call $D_{\mathrm{C}}\left(X_{N}\right):=D\left(\mathcal{F} ; X_{N}\right)$ the spherical cap discrepancy. 
Lemma 1.4. Let $d \geq 2$ and $X_{N}, N \geq 2$, be a sequence of $N$-point configurations on $\mathbb{S}^{d}$. Then the following assertions are equivalent:

(1) The configurations $X_{N}$ are asymptotically uniformly distributed as $N \rightarrow \infty$.

(2) $\lim _{N \rightarrow \infty} \mathrm{Q}_{N}[f]=\mathrm{I}[f]$ for every function $f$ continuous on $\mathbb{S}^{d}$.

(3) $\lim _{N \rightarrow \infty} \mathrm{Q}_{N}\left[Y_{\ell, m}\right]=0$ for every spherical harmonic $Y_{\ell, m}$ of degree $\ell \geq 1$ from an $\mathbb{L}^{2}\left(\mathbb{S}^{d}, \sigma\right)$-orthonormal basis $\left\{Y_{\ell, m}\right\}$.

(4) $\lim _{N \rightarrow \infty} D_{\mathrm{C}}\left(X_{N}\right)=0$.

(5) $\lim _{N \rightarrow \infty} \frac{\left|X_{N} \cap C\right|}{N}=\sigma(C)$ uniformly for all spherical caps $C$.

Remark 1.5. Property (3) is the spherical version of Weyl's criterion [18.

Lemma 1.4 can be proved by showing the sequence of implications $(1) \Rightarrow(2) \Rightarrow$ $(3) \Rightarrow(4) \Rightarrow(1)$. The implication $(2) \Rightarrow(3)$ uses the discrepancy estimate

$$
D_{\mathrm{C}}\left(X_{N}\right) \leq \frac{c_{1}}{L+1}+\sum_{\ell=1}^{L}\left(\frac{c_{2}}{\ell}+\frac{c_{3}}{L+1}\right) \sum_{m=1}^{Z(d, \ell)}\left|\frac{1}{N} \sum_{j=1}^{N} Y_{\ell, m}\left(\mathbf{x}_{j}\right)\right|
$$

following from a generalization of the well-known Erdös-Turán inequality [10] (see also [21]). The estimate above holds for any positive integer $L$; the positive constants $c_{1}, c_{2}$, and $c_{3}$ depend on $d$ only. The integers

$$
Z(d, 0)=1, \quad Z(d, \ell)=(2 \ell+d-1) \frac{\Gamma(\ell+d-1)}{\Gamma(d) \Gamma(\ell+1)}
$$

denote the number of linearly independent spherical harmonics of degree $\ell$.

Proposition 1. Let $d \geq 2$. Then optimal logarithmic energy $N$-point configurations are uniformly distributed as $N \rightarrow \infty$.

In Subsection 2.1 we include a proof of this result using a non-potential theoretical argument based on Lemma 1.4(3). It also follows from (1.10) and Lemma 1.4(4).

Proposition 1 is a qualitative result. It does not give a measure of how "good" optimal logarithmic energy points are uniformly distributed. Our next result quantifies uniform distribution of such point sets.

Theorem 1.6. Let $d \geq 2$. The spherical cap discrepancy of optimal logarithmic energy $N$-point configurations $X_{N}^{*}$ can be bounded by

$$
D_{\mathrm{C}}\left(X_{N}^{*}\right)=\mathcal{O}\left(N^{-1 /(d+2)}\right), \quad N \rightarrow \infty .
$$

The proof of Theorem 1.6 is given in Subsection 2.2 for more general, so-called $K$-regular, test sets. (See the definition there.)

Remark 1.7. Beck observed 3 that to any $N$-point set $X_{N}$ on the $d$-sphere there exists a spherical cap $C$ such that

$$
c_{1} N^{-1 / 2-1 /(2 d)}<\left|\frac{\left|X_{N} \cap C\right|}{N}-\sigma(C)\right|
$$

and from probability arguments (see [3]) there follows that there exist $N$-point sets $X_{N}$ on $\mathbb{S}^{d}$ such that

$$
\left|\frac{\left|X_{N} \cap C\right|}{N}-\sigma(C)\right|<c_{2} N^{-1 / 2-1 /(2 d)} \sqrt{\log N}
$$


for any spherical cap $C$. $\left(c_{i}>0\right.$ are constants independent of $N$.) For optimal Riesz $(d-1)$-energy points Korevaar [16] conjectured that $D_{\mathrm{C}}\left(X_{N}^{*, d-1}\right)=\mathcal{O}\left(N^{-1 / d}\right)$. Götz [9] proved this up to a logarithmic factor. Götz also showed a lower bound of order $N^{-1 / 2}$ on $\mathbb{S}^{2}$.

As a by-product of the proof of Theorem 1.6 we obtain estimates for the error of integration for certain classes of functions defined on $\mathbb{S}^{d}$.

Corollary 1.8. Let $d \geq 2$. Then equally-weighted numerical integration rules $\mathrm{Q}_{N}$ with nodes forming optimal logarithmic energy configurations satisfy

$$
\left|\mathrm{Q}_{N}[p]-\mathrm{I}[p]\right| \leq C_{d}\left(N^{1 / d} / n\right)^{-d / 2}\|p\|_{\infty}, \quad \text { as } n / N^{1 / d} \rightarrow 0,
$$

for all polynomials $p$ on $\mathbb{S}^{d}$ of degree $\leq n$. The positive constant $C_{d}$ does not depend on $n, N$, or $p$.

Using [6. Theorem 2], we can obtain bounds for the error of integrations for continuous functions of $\mathbb{S}^{d}$ satisfying a Lipschitz condition:

Corollary 1.9. Let $d \geq 2$. Let $f$ be a continuous function of $\mathbb{S}^{d}$ with

$$
|f(\mathbf{x})-f(\mathbf{y})| \leq C_{f} \arccos (\langle\mathbf{x}, \mathbf{y}\rangle), \quad \mathbf{x}, \mathbf{y} \in \mathbb{S}^{d} .
$$

Then equally-weighted numerical integration rules $\mathrm{Q}_{N}$ with nodes forming optimal logarithmic energy configurations satisfy

$$
\left|\mathrm{Q}_{N}[f]-\mathrm{I}[f]\right| \leq\left(12 d C_{f}+C_{d}^{\prime}\|f\|_{\infty}\right) \mathcal{O}\left(N^{-1 /(d+2)}\right), \quad N \rightarrow \infty .
$$

For other results concerning the error of integration for numerical integration formulas on $\mathbb{S}^{d}$ we refer to the literature (for example [5, 12, 13, 14, 15]).

\section{Proofs AND Discussions}

In this section we collect auxiliary results and proofs of our lemmas and theorems. Furthermore, we discuss technical aspects.

2.1. Preliminaries and proof of Proposition 1. The distance of two points on the unit sphere can be expressed as $|\mathbf{x}-\mathbf{y}|^{2}=2(1-\langle\mathbf{x}, \mathbf{y}\rangle)$. Hence, the logarithmic kernel $k(\mathbf{x}):=\log (1 /|\mathbf{x}|)$ takes the form

$$
k(\mathbf{x}-\mathbf{y})=-\frac{1}{2} \log 2-\frac{1}{2} \log (1-\langle\mathbf{x}, \mathbf{y}\rangle) .
$$

To avoid the singularity at $\mathbf{x}=\mathbf{y}$ a $\delta$-kernel can be defined as

$$
k^{\delta}(\mathbf{x}-\mathbf{y}):=-\frac{1}{2} \log 2-\frac{1}{2} \log (1+2 \delta-\langle\mathbf{x}, \mathbf{y}\rangle), \quad 0<\delta<1 .
$$

The $\delta$-kernel can be expanded in a series of Gegenbauer polynomials $P_{n}$ associated with the $d$-sphere, normalized such that $P_{n}(1)=1$ :

$$
k^{\delta}(\mathbf{x}-\mathbf{y})=\sum_{n=0}^{\infty} \frac{a_{n}(\delta)}{Z(d, n)} Z(d, n) P_{n}(\langle\mathbf{x}, \mathbf{y}\rangle),
$$


where the series coefficients are given in terms of the modified energy integral and in terms of hypergeometric functions (see Subsection 2.5)

$$
\begin{aligned}
\frac{a_{0}(\delta)}{Z(d, 0)} & =\mathcal{I}^{\delta}[\sigma], \\
\frac{a_{n}(\delta)}{Z(d, n)} & =\frac{1}{2} \frac{(n-1) !(d / 2)_{n}}{(d)_{2 n}}\left(\frac{1}{1+\delta}\right)^{n}{ }_{2} \mathrm{~F}_{1}\left(\begin{array}{c}
n, n+d / 2 \\
2 n+d
\end{array} \frac{1}{1+\delta}\right) .
\end{aligned}
$$

$(a)_{n}$ is the Pochhammer symbol. The coefficients are strictly monotonically increasing as $\delta \rightarrow 0$. Hence, by [1, 15.1.20],

$$
a_{n}(\delta) \leq a_{n}(0)\left(\frac{1}{1+\delta}\right)^{n}=\frac{\Gamma(d)}{2} \frac{\Gamma(n)}{\Gamma(n+d)}\left(\frac{1}{1+\delta}\right)^{n} .
$$

So, the series expansion (2.2) is uniformly convergent in $\mathbf{x}, \mathbf{y} \in \mathbb{S}^{d}$ for every fixed $0<\delta<1$.

Including the now well defined diagonal terms we can write

$$
\begin{gathered}
\frac{E^{\delta}\left(X_{N}\right)}{N^{2}}+\frac{\log (4 \delta)^{-1 / 2}}{N}=\sum_{n=0}^{\infty} a_{n}(\delta)\left(\frac{1}{N^{2}} \sum_{j, k} P_{n}\left(\left\langle\mathbf{x}_{j}, \mathbf{x}_{k}\right\rangle\right)\right) \\
\geq a_{0}(\delta)+a_{n}(\delta)\left(\frac{1}{N^{2}} \sum_{j, k} P_{n}\left(\left\langle\mathbf{x}_{j}, \mathbf{x}_{k}\right\rangle\right)\right) \geq 0, \quad n \geq 1 .
\end{gathered}
$$

The non-negativity follows from the positivity of the series coefficients and the addition theorem for spherical harmonics, that is,

$$
\frac{1}{N^{2}} \sum_{j, k} Z(d, n) P_{n}\left(\left\langle\mathbf{x}_{j}, \mathbf{x}_{k}\right\rangle\right)=\sum_{m=1}^{Z(d, n)}\left|\frac{1}{N} \sum_{j=1}^{N} Y_{n, m}\left(\mathbf{x}_{j}\right)\right|^{2} .
$$

Combining both (2.3) and (2.4) and using $E^{\delta}\left(X_{N}\right) \leq E\left(X_{N}\right)$ we obtain

$$
\begin{aligned}
\frac{a_{n}(\delta)}{Z(d, n)}\left|\frac{1}{N} \sum_{j=1}^{N} Y_{n, m}\left(\mathbf{x}_{j}\right)\right|^{2} \\
\quad \leq\left(\frac{E\left(X_{N}\right)}{N^{2}}-\mathcal{I}[\sigma]\right)+\frac{\log (4 \delta)^{-1 / 2}}{N}+\left(\mathcal{I}[\sigma]-a_{0}(\delta)\right) .
\end{aligned}
$$

This inequality holds for every $0<\delta<1$, in particular for $4 \delta=N^{-2 / d}$. If a sequence $X_{N}, N \geq 2$, consists of optimal logarithmic energy point configurations, all three terms on the right-hand side above tend to 0 as $N \rightarrow \infty$. This follows from (1.5), $\log (4 \delta)^{-1 / 2} / N=(1 / d)(\log N) / N$, and $\lim _{\delta \rightarrow 0} a_{0}(\delta)=\mathcal{I}[\sigma]$. Thus, Property (3) in Lemma 1.4 is satisfied. Hence, optimal logarithmic energy points are uniformly distributed.

2.2. Quantification of uniform distribution. We want to obtain an estimate for the discrepancy of an $N$-point set in terms of its logarithmic energy. The family of test sets $\mathcal{F}=\mathcal{F}_{K}, K>0$ fixed, may consist of all $K$-regular sets in $\mathbb{S}^{d}$. A $\sigma$ measurable set $B \subseteq \mathbb{S}^{d}$ is defined to be $K$-regular if its $\delta$-neighborhood ( $\delta$ sufficiently small) is linearly bounded in $\delta$, that is, $\sigma\left(\partial_{\delta}^{\mathbb{S}^{d}} B\right) \leq K \delta, \delta>0$, where

$$
\partial_{\delta}^{\mathbb{S}^{d}} B:=\left\{\mathbf{x} \in \mathbb{S}^{d} \mid \operatorname{dist}(B, \mathbf{x}) \leq \delta, \operatorname{dist}\left(\mathbb{S}^{d} \backslash B, \mathbf{x}\right) \leq \delta\right\},
$$


and $\operatorname{dist}(A, \mathbf{x})$ denotes the Euclidean distance of a point $\mathbf{x}$ from a set $A$ and $\operatorname{dist}(\emptyset, \mathbf{x}):=\infty$. Such sets were introduced by Sjögren [28]. Clearly, spherical caps are $K$-regular for some $K>0$.

[2, Theorem 1] gives a relation between discrepancy and the quality of the corresponding equally weighted quadrature formula for polynomials. There exists a number $C_{0}>0$ depending only on $d$ such that every $K$-regular set $B \subseteq \mathbb{S}^{d}$ satisfies

$$
\left|\frac{\left|X_{N} \cap B\right|}{N}-\sigma(B)\right| \leq C_{0} \inf _{m \in \mathbb{N}}\left\{\frac{K}{m}+C_{q m}\left(d ; X_{N}\right)\right\},
$$

where

$$
C_{q m}\left(d ; X_{N}\right):=\sup \left\{\left|\mathrm{Q}_{N}[p]-\mathrm{I}[p]\right| \begin{array}{c}
p \text { polynomial on } \mathbb{S}^{d}, \\
\operatorname{deg} p \leq q m,\|p\|_{\infty} \leq 1
\end{array}\right\}
$$

and $q$ is the smallest integer satisfying $2 q \geq d+31$

The error of integration, $R\left(p ; X_{N}\right):=\mathrm{Q}_{N}[p]-\mathrm{I}[p]$, can be bounded in terms of the energy of $X_{N}$. For a polynomial $p$ on $\mathbb{S}^{d}$ of degree at most $n \geq 1$ one has [6]

$$
\left|R\left(p ; X_{N}\right)\right| \leq \sqrt{\frac{E^{\delta}\left(X_{N}\right) / N^{2}-a_{0}(\delta)+g^{\delta}(1) / N}{\min _{1 \leq k \leq n} a_{k}(\delta) / Z(d, k)}}\|p\|_{\infty} .
$$

All together this gives an upper estimate for the discrepancy

$$
D\left(\mathcal{F}_{K} ; X_{N}\right) \leq C_{0}\left\{\frac{K}{m}+\sqrt{\frac{E^{\delta}\left(X_{N}\right) / N^{2}-a_{0}(\delta)+g^{\delta}(1) / N}{\min _{1 \leq k \leq q m} a_{k}(\delta) / Z(d, k)}}\right\}
$$

which holds for any $m \in \mathbb{N}$, any $0<\delta<\delta_{0}$ and any $N$-point-sets $X_{N}$.

First we focus on the numerator under the root sign in (2.5), that is,

$$
E^{\delta}\left(X_{N}\right) / N^{2}-a_{0}(\delta)+\left[\log (4 \delta)^{-1 / 2}\right] / N .
$$

We use (2.15) to estimate the modified energy $E^{\delta}\left(X_{N}\right)$ in (2.6). From $N^{2} a_{0}(\delta)=$ $N(N-1) a_{0}(\delta)+N a_{0}(\delta)$ and (2.16)

$$
\frac{E^{\delta}\left(X_{N}\right)}{N^{2}}-a_{0}(\delta)+\frac{\log (4 \delta)^{-1 / 2}}{N} \leq \frac{E\left(X_{N}\right)-\underline{E}_{N}}{N^{2}}-\frac{a_{0}(\delta)}{N}
$$

where $\underline{E}_{N}$ is the right-hand side in (2.17). By (2.17), $\underline{E}_{N}$ is a lower bound for $E\left(X_{N}\right)$. In Subsection 2.4 we estimate $\underline{E}_{N}$ from below to get a lower bound for the optimal logarithmic energy. For minimum logarithmic energy point sets $X_{N}$ the expression $\left(E\left(X_{N}\right)-\underline{E}_{N}\right) / N^{2}$ can be estimated from above by the difference of upper (1.5) and lower bound (Lemma 1.1) for $E\left(X_{N}\right)$. We chose $4 \delta=N^{-2 / d}$. From (2.24) and (2.25) (using residue calculus) one gets $a_{0}(\delta)=\mathcal{I}[\sigma]-\mathcal{O}\left(N^{-2 / d}\right)$ as $N \rightarrow \infty$ for $d \geq 2$. Hence

$$
\frac{E^{\delta}\left(X_{N}\right)}{N^{2}}-a_{0}(\delta)+\frac{\log (4 \delta)^{-1 / 2}}{N} \leq \frac{c_{2}+C_{d}^{\prime}-\mathcal{I}[\sigma]}{N}+\mathcal{O}\left(N^{-1-2 \varepsilon / d}\right),
$$

as $N \rightarrow \infty$. For $d \geq 2$ the lower bound of $\underline{E}_{N}$ is given in Lemma 1.1 .

The denominator under the root sign in (2.5) can be replaced by $a_{n}(\delta) / Z(d, n)$ for any $n \geq q m$, since the sequence $a_{k}(\delta) / Z(d, k), k \geq 1$, is strictly monotonically

\footnotetext{
${ }^{1}$ Note that in 2] everything is done in $\mathbb{R}^{d}$.
} 
decreasing as $k \rightarrow \infty$ (Lemma 2.2). The asymptotics of $a_{k}(\delta) / Z(d, k)$ is given in Lemma 2.3. Thus

$$
D\left(\mathcal{F}_{K} ; X_{N}\right) \leq C_{0}\left\{\frac{K}{m}+\sqrt{\frac{2\left(c_{2}+C_{d}^{\prime}-\mathcal{I}[\sigma]\right) N^{-1} n^{d}\left\{1+\mathcal{O}\left(N^{-1 / d}\right)\right\}}{\Gamma(d / 2)\{1+\mathcal{O}(1 / n)\}\left\{1+\mathcal{O}\left(n^{2 \varepsilon} / N^{2 \varepsilon / d}\right)\right\}}}\right\} .
$$

We used [1, 6.1.47]. Let $n=q m$. Assume $m=N^{\alpha / d}$. The constant $\alpha$ is chosen to balance both contributions to the discrepancy, that is,

$$
N^{-\alpha / d}=m^{-1} \asymp \sqrt{N^{-1} m^{d}}=N^{(\alpha-1) / 2}, \quad N \rightarrow \infty .
$$

The asymptotical relation above is solved by $\alpha=d /(d+2)$. Note, $\mathcal{O}\left(n^{2 \varepsilon} N^{-2 \varepsilon / d}\right)=$ $\mathcal{O}\left(N^{-4 \varepsilon /[d(d+2)]}\right)$. This finishes the proof of Theorem [1.6.

2.3. Representing the logarithmic energy through modified energies. We consider the Taylor expansion of $g^{\delta}$ for small $\delta$, that is,

$$
g^{\delta}=\left.\sum_{k=0}^{K} \frac{\mathrm{d}^{k} g^{\delta}}{\mathrm{d} \delta^{k}}\right|_{0} \frac{\delta^{k}}{k !}+\left.\frac{\delta^{K+1}}{K !} \int_{0}^{1}(1-\theta)^{K} \frac{\mathrm{d}^{K+1} g^{\delta}}{\mathrm{d} \delta^{K+1}}\right|_{\theta \delta} \mathrm{d} \theta
$$

The derivatives of $g^{\delta}(t)$ with respect to $\delta$, where $g^{\delta}(\langle\mathbf{x}, \mathbf{y}\rangle):=k^{\delta}(\mathbf{x}-\mathbf{y})$, are

$$
\left(g^{\delta}\right)^{(n)}(t)=(-1)^{n} 2^{n-1}(n-1) !(1+2 \delta-t)^{-n}, \quad n \geq 1 .
$$

Then the logarithmic energy of an $N$-point set $X_{N}$ takes on the form

$$
E^{\delta}\left(X_{N}\right)=E\left(X_{N}\right)+\frac{1}{2} \sum_{k=1}^{K} \frac{(-1)^{k}}{k}(4 \delta)^{k} E_{2 k}\left(X_{N}\right)+\mathrm{R}_{K}(\delta),
$$

where $E_{s}\left(X_{N}\right)$ is the Riesz s-energy of $X_{N}$. The remainder term is given by

$$
\mathrm{R}_{K}(\delta):=\frac{(-1)^{K+1}}{2}(4 \delta)^{K+1} \int_{0}^{1}(1-\theta)^{K} E_{2(K+1)}^{\theta \delta}\left(X_{N}\right) \mathrm{d} \theta .
$$

In 4 we express the Riesz $s$-energy in terms of modified Riesz energies. Recall that the related $\delta$-kernel is given by $k_{s}^{\delta}(\mathbf{x})=\left(4 \delta+|\mathbf{x}|^{2}\right)^{-s / 2}$. We have

$$
E_{s}\left(X_{N}\right)=\sum_{\ell=0}^{L} \frac{(s / 2)_{\ell}}{\ell !}(4 \delta)^{\ell} E_{s+2 \ell}^{\delta}\left(X_{N}\right)+\mathrm{R}_{L}(s ; \delta),
$$

where the remainder term reads as

$$
\mathrm{R}_{L}(s ; \delta):=\frac{(s / 2)_{L+1}}{L !}(4 \delta)^{L+1} \int_{0}^{1} \theta^{L} E_{s+2 L+2}^{\theta \delta}\left(X_{N}\right) \mathrm{d} \theta .
$$

Substitute (2.11) into (2.9) such that the modified energy under the integral sign in the remainder term is always of the form $E_{2(K+1)}^{\theta \delta}\left(X_{N}\right)$ :

$$
\begin{aligned}
E^{\delta}\left(X_{N}\right)=E & \left(X_{N}\right)+\frac{1}{2} \sum_{k=1}^{K} \sum_{\ell=0}^{K-k}(-1)^{k} \frac{(k)_{\ell}}{k \ell !}(4 \delta)^{k+\ell} E_{2(k+\ell)}^{\delta}\left(X_{N}\right) \\
& +\frac{1}{2} \sum_{k=1}^{K} \frac{(-1)^{k}}{k}(4 \delta)^{k} \mathrm{R}_{K-k}(2 k ; \delta)+\mathrm{R}_{K}(\delta) .
\end{aligned}
$$


Reorder the double sum with respect to $r=k+\ell, r=1, \ldots, K$. Then, by the Binomial Theorem, the new inner sum is

$$
\sum_{\ell=0}^{r-1} \frac{(r-\ell)_{\ell}}{(r-\ell) \ell !}(-1)^{r-\ell}=\frac{1}{r} \sum_{\ell=0}^{r-1}\left(\begin{array}{l}
r \\
\ell
\end{array}\right)(-1)^{r-\ell}=-\frac{1}{r} .
$$

For the combined remainder terms in (2.13) we get

$$
\frac{1}{2}(4 \delta)^{K+1} \int_{0}^{1}\left[f(\theta)+(-1)^{K+1}(1-\theta)^{K}\right] E_{2(K+1)}^{\theta \delta}\left(X_{N}\right) \mathrm{d} \theta
$$

where

$$
f(\theta)=\sum_{k=1}^{K} \frac{\Gamma(K+1)}{k \Gamma(k)(K-k) !}(-1)^{k} \theta^{K-k}=(\theta-1)^{K}-\theta^{K} .
$$

All together this gives

$$
\begin{aligned}
E\left(X_{N}\right)= & E^{\delta}\left(X_{N}\right)+\frac{1}{2} \sum_{r=1}^{K} \frac{1}{r}(4 \delta)^{r} E_{2 r}^{\delta}\left(X_{N}\right) \\
& +\frac{1}{2}(4 \delta)^{K+1} \int_{0}^{1} \theta^{K} E_{2(K+1)}^{\theta \delta}\left(X_{N}\right) \mathrm{d} \theta .
\end{aligned}
$$

2.4. Improving the lower bound for the optimal logarithmic energy. We observe that each term at the right-hand side of (2.14) is positive. From

$$
E_{2(K+1)}^{\theta \delta}\left(X_{N}\right) \geq E_{2(K+1)}^{\delta}\left(X_{N}\right)
$$

there follows the lower bound

$$
E\left(X_{N}\right) \geq E^{\delta}\left(X_{N}\right)+\frac{1}{2} \sum_{r=1}^{K+1} \frac{1}{r}(4 \delta)^{r} E_{2 r}^{\delta}\left(X_{N}\right)
$$

From (2.3), and a similar relation holding for the modified Riesz $s$-energy, we get the lower estimates

$$
\begin{aligned}
& E^{\delta}\left(X_{N}\right) \geq N(N-1) a_{0}(\delta)-N \log (4 \delta)^{-1 / 2}, \\
& E_{2 r}^{\delta}\left(X_{N}\right) \geq N(N-1) a_{0}(2 r ; \delta)-N(4 \delta)^{-r} .
\end{aligned}
$$

All together this gives us a lower bound for the discrete logarithmic energy

$$
\begin{gathered}
E\left(X_{N}\right) \geq N(N-1) a_{0}(\delta)+N(N-1) \frac{1}{2} \sum_{r=1}^{K+1} \frac{1}{r}(4 \delta)^{r} a_{0}(2 r ; \delta) \\
-N \log (4 \delta)^{-1 / 2}-N \frac{1}{2} \sum_{r=1}^{K+1} \frac{1}{r}
\end{gathered}
$$

Lemma 2.1. Let $d=2,3,4,5,6,7, \cdots$. Then

$$
\frac{1}{N^{2}} E\left(X_{N}\right) \geq \mathcal{I}[\sigma]-\frac{1}{d} \frac{\log N}{N}-\frac{C_{d}^{\prime}}{N}+\mathcal{O}_{\varepsilon}\left(N^{-1-2 \varepsilon / d}\right), \quad N \rightarrow \infty,
$$

for any $N$-point set on $\mathbb{S}^{d}$, where

$$
C_{d}^{\prime}=\mathcal{I}[\sigma]+\frac{1}{d} \frac{\Gamma(d) \Gamma(1+\lfloor d / 2\rfloor-d / 2)}{2^{d} \Gamma(d / 2) \Gamma(1+\lfloor d / 2\rfloor)}+\frac{1}{2} \sum_{r=1}^{\lfloor d / 2\rfloor} \frac{1}{r}>0
$$

and $0<\varepsilon<1$ for even $d$ and $0<\varepsilon<1 / 2$ for odd $d$. 
Proof. Substituting (2.35) we obtain

$$
\begin{aligned}
& \frac{1}{2} \sum_{r=1}^{K+1} \frac{1}{r}(4 \delta)^{r} a_{0}(2 r ; \delta)=\frac{1}{2} \frac{\Gamma(d)}{\Gamma(d / 2)} \\
& \quad \times \frac{1}{2 \pi \mathrm{i}} \int_{-\mathrm{i} \infty}^{\mathrm{i} \infty}\left(\sum_{r=1}^{K+1} \frac{\Gamma(r+\zeta)}{r \Gamma(r)}\right) \frac{\Gamma(d / 2+\zeta) \Gamma(-\zeta)}{\Gamma(d+\zeta)}\left(\frac{1}{\delta}\right)^{\zeta} \mathrm{d} \zeta .
\end{aligned}
$$

It can be easily verified (using induction) that

$$
\sum_{r=1}^{K+1} \frac{\Gamma(r+\zeta)}{r \Gamma(r)}=-\Gamma(\zeta)+\frac{\Gamma(K+2+\zeta)}{(K+1) ! \zeta} .
$$

Now, observe that the contribution due to the term $-\Gamma(\zeta)$ is exactly the expression (2.25) which appears with a negative sign in (2.24). Thus, using (2.26), we have

$$
a_{0}(\delta)+\frac{1}{2} \sum_{r=1}^{K+1} \frac{1}{r}(4 \delta)^{r} a_{0}(2 r ; \delta)=\mathcal{I}[\sigma]+R(\delta) .
$$

The remainder term is

$$
\begin{aligned}
R(\delta) & :=\frac{1}{2} \frac{\Gamma(d)}{\Gamma(d / 2)(K+1) !} \\
& \times \frac{1}{2 \pi \mathrm{i}} \int_{-\mathrm{i} \infty}^{\mathrm{i} \infty} \frac{\Gamma(K+2+\zeta) \Gamma(d / 2+\zeta) \Gamma(-\zeta)}{\zeta \Gamma(d+\zeta)}\left(\frac{1}{\delta}\right)^{\zeta} \mathrm{d} \zeta .
\end{aligned}
$$

Next we consider the behavior of $R(\delta)$ as $\delta \rightarrow 0$. We set $K+1=\lfloor d / 2\rfloor$. Here $\lfloor x\rfloor$ denotes the largest integer $\leq x$. The integrand in (2.20) has its first pole to the left of the contour at $\zeta=-d / 2$ with residue

$$
-\frac{2}{d} \Gamma(1+\lfloor d / 2\rfloor-d / 2) \delta^{d / 2}
$$

Moving the line of integration over the pole at $\zeta=-d / 2$ residue calculus yields

$$
\begin{aligned}
R(\delta)= & -\frac{1}{d} \frac{\Gamma(d) \Gamma(1+\lfloor d / 2\rfloor-d / 2)}{\Gamma(d / 2) \Gamma(1+\lfloor d / 2\rfloor)} \delta^{d / 2} \\
& +\frac{1}{2} \frac{\Gamma(d)}{\Gamma(d / 2) \Gamma(1+\lfloor d / 2\rfloor)} \delta^{d / 2+\varepsilon} \frac{1}{2 \pi \mathrm{i}} \int_{-\infty}^{\infty} H(\mathrm{i} t) \mathrm{d} t,
\end{aligned}
$$

where

$$
H(\zeta):=\frac{\Gamma(1+\lfloor d / 2\rfloor-d / 2-\varepsilon+\zeta) \Gamma(-\varepsilon+\zeta) \Gamma(d / 2+\varepsilon-\zeta)}{(-d / 2-\varepsilon+\zeta) \Gamma(d / 2-\varepsilon+\zeta)}\left(\frac{1}{\delta}\right)^{\zeta} .
$$

The number $\varepsilon$ needs to be chosen such that the line of integration passes the real axis between the poles at $\zeta=-d / 2$ and the next pole at $\zeta=-1-\lfloor d / 2\rfloor$. That is, $0<\varepsilon<1$ for dimension $d$ is even and $0<\varepsilon<1 / 2$ for $d$ is odd. By Stirling's formula the integral can be bounded by a constant depending on $\varepsilon$ and $d$ only. Thus

$$
R(\delta)=-\frac{1}{d} \frac{\Gamma(d) \Gamma(1+\lfloor d / 2\rfloor-d / 2)}{\Gamma(d / 2) \Gamma(1+\lfloor d / 2\rfloor)} \delta^{d / 2}+\mathcal{O}_{\varepsilon}\left(\delta^{d / 2+\varepsilon}\right), \quad \delta \rightarrow 0 .
$$

We choose $4 \delta=N^{-2 / d}$ and substitute (2.19) and (2.21) into (2.17). 
2.5. Expanding $k^{\delta}$ in a series of Gegenbauer polynomials. The series coefficients in (2.2) can be calculated using the formula (see [22])

$$
\frac{a_{n}(\delta)}{Z(d, n)}=\frac{\omega_{d-1}}{\omega_{d}} \int_{-1}^{1} g^{\delta}(t) P_{n}(t)\left(1-t^{2}\right)^{d / 2-1} \mathrm{~d} t
$$

where $g^{\delta}(\langle\mathbf{x}, \mathbf{y}\rangle):=k^{\delta}(\mathbf{x}-\mathbf{y}), \omega_{d}$ denotes the surface area of $\mathbb{S}^{d}$, and

$$
\frac{\omega_{d}}{\omega_{d-1}}=\int_{-1}^{1}\left(1-t^{2}\right)^{d / 2-1} \mathrm{~d} t=\frac{\sqrt{\pi} \Gamma(d / 2)}{\Gamma((d+1) / 2)}=2^{d-1} \frac{[\Gamma(d / 2)]^{2}}{\Gamma(d)} .
$$

From (2.1),$P_{0}(t) \equiv 1$, and (2.23) we get

$$
\frac{a_{0}(\delta)}{Z(d, 0)}=-\frac{1}{2} \log 2-\frac{1}{2} \frac{\omega_{d-1}}{\omega_{d}} \int_{-1}^{1} \log (1+2 \delta-t)\left(1-t^{2}\right)^{d / 2-1} \mathrm{~d} t
$$

A change of variable $2 u=1-t$ leads to

$$
\frac{a_{0}(\delta)}{Z(d, 0)}=-\frac{1}{2} \log 2-\frac{1}{2} \frac{\omega_{d-1}}{\omega_{d}} 2^{d-1} \int_{0}^{1} u^{d / 2-1}(1-u)^{d / 2-1} \log (2 \delta+2 u) \mathrm{d} u .
$$

By [24, 2.6.10(31)] the last integral evaluates as

$$
\frac{\Gamma(d / 2) \Gamma(d / 2)}{\Gamma(d)} \log (2 \delta)+\delta^{-1} \frac{\Gamma(1+d / 2) \Gamma(d / 2)}{\Gamma(d+1)}{ }_{3} \mathrm{~F}_{2}\left(\begin{array}{c}
1+d / 2,1,1 \\
d+1,2
\end{array} ;-\frac{1}{\delta}\right) .
$$

Using (2.23) we get

$$
\begin{aligned}
\frac{a_{0}(\delta)}{Z(d, 0)}=- & \log 2-\frac{1}{2} \log \delta-\frac{1}{2} \frac{\omega_{d-1}}{\omega_{d}} 2^{d-1} \delta^{-1} \\
& \times \frac{\Gamma(1+d / 2) \Gamma(d / 2)}{\Gamma(d+1)}{ }_{3} \mathrm{~F}_{2}\left(\begin{array}{c}
1+d / 2,1,1 \\
d+1,2
\end{array} ;-\frac{1}{\delta}\right) .
\end{aligned}
$$

Using [25, Thm. 36], we have

$$
\frac{a_{0}(\delta)}{Z(d, 0)}=-\log 2-\frac{1}{2} \log \delta-\frac{1}{2} \frac{\omega_{d-1}}{\omega_{d}} 2^{d-1} \delta^{-1} \Gamma(d / 2) I_{0}(\delta),
$$

where $I_{0}(\delta)$ denotes the Mellin-Barnes integral

$$
I_{0}(\delta):=\frac{1}{2 \pi \mathrm{i}} \int_{-\mathrm{i} \infty}^{\mathrm{i} \infty} \frac{\Gamma(1+d / 2+\zeta)[\Gamma(1+\zeta)]^{2} \Gamma(-\zeta)}{\Gamma(2+\zeta) \Gamma(d+1+\zeta)}\left(\frac{1}{\delta}\right)^{\zeta} \mathrm{d} \zeta .
$$

The contour is chosen such that the poles of $\Gamma(1+d / 2+\zeta)$ and $\Gamma(1+\zeta)$ are at its left side and the poles of $\Gamma(-\zeta)$ are at its right side. The path of integration is moved to the left over the pole at $\zeta=-1$. The integrand has a double pole at $\zeta=-1$ with residuum

$$
-\frac{\Gamma(d / 2)}{\Gamma(d)}[\psi(d)-\psi(d / 2)+\log \delta] \delta,
$$

where $\psi(z)$ denotes the Digamma function. Thus, using (2.23), we get

$$
\frac{a_{0}(\delta)}{Z(d, 0)}=-\log 2+\frac{1}{2}[\psi(d)-\psi(d / 2)]-R_{0}(\delta),
$$

where $R_{0}(\delta)$ denotes the remainder term

$$
R_{0}(\delta)=\frac{1}{2} \frac{\Gamma(d)}{\Gamma(d / 2)} \frac{1}{2 \pi \mathrm{i}} \int_{-\mathrm{i} \infty}^{\mathrm{i} \infty} \frac{\Gamma(d / 2+\zeta) \Gamma(\zeta) \Gamma(1-\zeta)}{\zeta \Gamma(d+\zeta)}\left(\frac{1}{\delta}\right)^{\zeta} \mathrm{d} \zeta .
$$


Observe that the 0-th coefficient is also the energy $\mathcal{I}^{\delta}[\sigma]$. By [24, 2.6.10(25)]

$$
\mathcal{I}[\sigma]=\frac{a_{0}(0)}{Z(d, 0)}=\lim _{\delta \rightarrow 0} \frac{a_{0}(\delta)}{Z(d, 0)}=-\log 2+\frac{1}{2}[\psi(d)-\psi(d / 2)] .
$$

Let $n \geq 1$. Rodrigues' formula yields

$$
\frac{a_{n}(\delta)}{Z(d, n)}=\frac{(-1)^{n}}{2^{n}(d / 2)_{n}} \frac{\omega_{d-1}}{\omega_{d}} \int_{-1}^{1} g^{\delta}(t)\left[\left(1-t^{2}\right)^{n+d / 2-1}\right]^{(n)} \mathrm{d} t .
$$

Apply $n$-times integration by parts. All boundary terms disappear. So

$$
\frac{a_{n}(\delta)}{Z(d, n)}=\frac{1}{2^{n}(d / 2)_{n}} \frac{\omega_{d-1}}{\omega_{d}} \int_{-1}^{1}\left[g^{\delta}\right]^{(n)}(t)\left(1-t^{2}\right)^{n+d / 2-1} \mathrm{~d} t .
$$

Since $\left[g^{\delta}\right]^{(n)}(t)=(1 / 2)(n-1) !(1+2 \delta-t)^{-n}, n \geq 1$, we have

$$
\frac{a_{n}(\delta)}{Z(d, n)}=\frac{(n-1) !}{2^{n+1}(d / 2)_{n}} \frac{\omega_{d-1}}{\omega_{d}} \int_{-1}^{1} \frac{\left(1-t^{2}\right)^{n+d / 2-1}}{(1+2 \delta-t)^{n}} \mathrm{~d} t .
$$

A change of variables $2 u=1+t$ gives

$$
\begin{aligned}
\frac{a_{n}(\delta)}{Z(d, n)}= & \frac{(n-1) !}{2(d / 2)_{n}} 2^{d-1} \frac{\omega_{d-1}}{\omega_{d}}\left(\frac{1}{1+\delta}\right)^{n} \\
& \quad \times \int_{0}^{1} u^{n+d / 2-1}(1-u)^{n+d / 2-1}\left(1-\frac{u}{1+\delta}\right)^{-n} \mathrm{~d} u .
\end{aligned}
$$

The right-most integral represents a hypergeometric function [1, 15.3.1]. Using Pochhammer symbols and (2.23), we obtain

$$
\frac{a_{n}(\delta)}{Z(d, n)}=\frac{(n-1) !(d / 2)_{n}}{2(d)_{2 n}}\left(\frac{1}{1+\delta}\right)^{n}{ }_{2} \mathrm{~F}_{1}\left(\begin{array}{c}
n, n+d / 2 \\
2 n+d
\end{array} ; \frac{1}{1+\delta}\right),
$$

or, using the series expansion of a hypergeometric function, we have

$$
\frac{a_{n}(\delta)}{Z(d, n)}=\frac{\Gamma(d)}{2 \Gamma(d / 2)} \sum_{m=0}^{\infty} \frac{\Gamma(m+n) \Gamma(m+n+d / 2)}{\Gamma(m+2 n+d) m !}\left(\frac{1}{1+\delta}\right)^{m+n},
$$

valid for all $\delta \geq 0$ and $n \geq 1$.

2.6. Monotonicity of the coefficients $a_{n}(\delta) / Z(d, n)$ as $n \rightarrow \infty$.

Lemma 2.2. Fix $0<\delta<1$. The sequence $a_{n}(\delta) / Z(d, n), n \geq 1$, is strictly monotonically decreasing as $n$ grows.

Proof. Define

$$
H(x):=\frac{\Gamma(m+x) \Gamma(m+x+d / 2)}{\Gamma(m+2 x+d)}, \quad x \geq 1 / 2 .
$$

Since the Digamma function is strictly monotonically increasing,

$$
\begin{aligned}
\frac{\mathrm{d} H}{\mathrm{~d} x}=- & H(x)\{[\psi(2 x+m+d)-\psi(x+m+d / 2)] \\
& +[\psi(2 x+m+d)-\psi(x+m)]\}
\end{aligned}
$$

is negative. Thus, the function $H$ is strictly monotonically decreasing. So, each term in the series expansion (2.29) is, as a product of two strictly decreasing functions, strictly monotonically decreasing. 
2.7. Asymptotics for $a_{n}(\delta) / Z(d, n)$ as $n \rightarrow \infty$. A change of variables $2 u=1-t$ in (2.27) leads to

$$
\frac{a_{n}(\delta)}{Z(d, n)}=\frac{(n-1) !(d / 2)_{n}}{2(d)_{2 n}}\left(\frac{1}{\delta}\right)^{n}{ }_{2} \mathrm{~F}_{1}\left(\begin{array}{c}
n, n+d / 2 \\
2 n+d
\end{array} ;-\frac{1}{\delta}\right) .
$$

Now, we use the Mellin-Barnes integral form [1, 15.3.2] to get

$$
\begin{aligned}
& \frac{a_{n}(\delta)}{Z(d, n)}=\frac{\Gamma(d)}{2 \Gamma(d / 2)}\left(\frac{1}{\delta}\right)^{n} \\
& \quad \times \frac{1}{2 \pi \mathrm{i}} \int_{-\mathrm{i} \infty}^{\mathrm{i} \infty} \frac{\Gamma(n+\zeta) \Gamma(n+d / 2+\zeta) \Gamma(-\zeta)}{\Gamma(2 n+d+\zeta)}\left(\frac{1}{\delta}\right)^{\zeta} \mathrm{d} \zeta .
\end{aligned}
$$

The contour is chosen such that the poles of $\Gamma(n+\zeta)$ and $\Gamma(n+d / 2+\zeta)$ are at its left side and the poles of $\Gamma(-\zeta)$ are at its right side. Moving the contour over the pole at $\zeta=-n$, residue calculus yields

$$
\begin{aligned}
& \frac{a_{n}(\delta)}{Z(d, n)}=\frac{\Gamma(d)}{2} \frac{\Gamma(n)}{\Gamma(n+d)}+\frac{\Gamma(d)}{2 \Gamma(d / 2)} \delta^{\varepsilon} \\
& \quad \times \frac{1}{2 \pi \mathrm{i}} \int_{-\mathrm{i} \infty}^{\mathrm{i} \infty} \frac{\Gamma(\zeta-\varepsilon) \Gamma(d / 2-\varepsilon+\zeta) \Gamma(n+\varepsilon-\zeta)}{\Gamma(n+d-\varepsilon+\zeta)}\left(\frac{1}{\delta}\right)^{\zeta} \mathrm{d} \zeta,
\end{aligned}
$$

$0<\varepsilon<1$. By Stirling's formula the integral is bounded. For our purpose this is not enough. The remainder term can be written as

$$
\frac{\Gamma(d)}{2} \frac{\Gamma(1-\varepsilon) \Gamma(d / 2-\varepsilon) \Gamma(n+\varepsilon)}{\Gamma(d / 2) \Gamma(n+d-\varepsilon)} \delta^{\varepsilon} \frac{1}{2 \pi \mathrm{i}} \int_{-\mathrm{i} \infty}^{\mathrm{i} \infty} \frac{\mathcal{L}(\zeta)}{\zeta-\varepsilon}\left(\frac{1}{\delta}\right)^{\zeta} \mathrm{d} \zeta
$$

$0<\delta<1$, where

$$
\mathcal{L}(\mathrm{i} t):=\frac{\Gamma(1-\varepsilon+\mathrm{i} t)}{\Gamma(1-\varepsilon)} \frac{\Gamma(d / 2-\varepsilon+\mathrm{i} t)}{\Gamma(d / 2-\varepsilon)} \frac{\Gamma(n+\varepsilon-\mathrm{i} t)}{\Gamma(n+\varepsilon)} \frac{\Gamma(n+d-\varepsilon)}{\Gamma(n+d-\varepsilon+\mathrm{i} t)} .
$$

By [1, 6.1.25]

$$
\begin{aligned}
|\mathcal{L}(\mathrm{i} t)|^{2} \leq[ & \left.+t^{2} /(1-\varepsilon)\right]^{-1}\left[1+t^{2} /(d / 2-\varepsilon)\right]^{-1} \\
& \times \frac{\prod_{m=0}^{\infty}\left[1+t^{2} /(m+n+\varepsilon)\right]^{-1}}{\prod_{m=0}^{\infty}\left[1+t^{2} /(m+n+d-\varepsilon)\right]^{-1}} .
\end{aligned}
$$

Since $0<\varepsilon<1$ and $d \geq 2$, the ratio of infinite products can be bounded from above by 1 . Thus, the integral in (2.33) can be bounded by a constant depending on $\varepsilon$ and $d$. This shows:

\section{Lemma 2.3.}

$$
\frac{a_{n}(\delta)}{Z(d, n)}=\frac{\Gamma(d / 2)}{2} \frac{\Gamma(n)}{\Gamma(n+d)}\left\{1+\mathcal{O}_{\varepsilon}\left(n^{2 \varepsilon}(4 \delta)^{\varepsilon}\right)\right\}, \quad n \rightarrow \infty,
$$

for any fixed $0<\varepsilon<1$.

Remark 2.4. The $\mathcal{O}_{\varepsilon}$-constant does not depend on $\delta$. 
2.8. Expanding $k_{s}^{\delta}$ in a series of Gegenbauer polynomials. Here $k_{s}^{\delta}(\mathbf{x})=$ $\left(4 \delta+|\mathbf{x}|^{2}\right)^{-s / 2}$ is the $\delta$-kernel for the modified Riesz $s$-energy,

$$
k_{s}^{\delta}(\mathbf{x}-\mathbf{y})=\sum_{n=0}^{\infty} \frac{a_{n}(s ; \delta)}{Z(d, n)} Z(d, n) P_{n}(\langle\mathbf{x}, \mathbf{y}\rangle) .
$$

A similar computation as in Subsection 2.5 leads to

$$
a_{0}(2 r ; \delta)=\frac{\omega_{d-1}}{\omega_{d}} \int_{-1}^{1} \frac{\left(1-t^{2}\right)^{d / 2-1}}{[4 \delta+2(1-t)]^{r}} \mathrm{~d} t=(4 \delta)^{-r}{ }_{2} \mathrm{~F}_{1}\left(r, d / 2 ;-\frac{1}{\delta}\right) .
$$

Now, we use the Mellin-Barnes integral form [1, 15.3.2] to get

$$
\begin{aligned}
a_{0}(2 r ; \delta)=( & 4 \delta)^{-r} \frac{\Gamma(d)}{\Gamma(d / 2) \Gamma(r)} \\
& \times \frac{1}{2 \pi \mathrm{i}} \int_{-\mathrm{i} \infty}^{\mathrm{i} \infty} \frac{\Gamma(r+\zeta) \Gamma(d / 2+\zeta) \Gamma(-\zeta)}{\Gamma(d+\zeta)}\left(\frac{1}{\delta}\right)^{\zeta} \mathrm{d} \zeta,
\end{aligned}
$$

where we integrate along a Barnes path of integration separating the poles of $\Gamma(r+\zeta)$ and $\Gamma(d / 2+\zeta)$ at its left side from the poles of $\Gamma(-\zeta)$ at its right side.

\section{ACKNOWLEDGMENTS}

The author would like to thank an anonymous referee for many helpful suggestions.

\section{REFERENCES}

[1] M. Abramowitz and I.A. Stegun (eds.), Handbook of mathematical functions with formulas, graphs, and mathematical tables, Dover, 1970. MR0208797 (34:8606)

[2] V.V. Andrievskii, H.-P. Blatt, and M. Götz, Discrepancy estimates on the sphere, Monatsh. Math. 128 (1999), no. 3, 179-188. MR.1719364 (2000h:11080)

[3] J. Beck, Sums of distances between points on a sphere - an application of the theory of irregularities of distribution to discrete geometry, Mathematika 31 (1984), no. 1, 33-41. MR762175 (86d:52004)

[4] J.S. Brauchart, About the second term of the asymptotics for optimal Riesz energy on the sphere in the potential-theoretical case, Integral Transforms Spec. Funct. 17 (2006), no. 5, 321-328. MR2237493 (2007f:31011)

[5] J.S. Brauchart and K. Hesse, Numerical integration over spheres of arbitrary dimension, Constr. Approx. 25 (2007), no. 1, 41-71. MR2263736 (2007f:41025)

[6] S.B. Damelin and P.J. Grabner, Energy functionals, numerical integration and asymptotic equidistribution on the sphere, J. Complexity 19 (2003), no. 3, 231-246. MR.1984111 (2004e:41032)

[7] P.D. Dragnev, On the separation of logarithmic points on the sphere, Approximation theory, X (St. Louis, MO, 2001), Innov. Appl. Math., Vanderbilt Univ. Press, Nashville, TN, 2002, pp. 137-144. MR.1924855 (2003h:41020)

[8] P.D. Dragnev, D.A. Legg, and D.W. Townsend, Discrete logarithmic energy on the sphere, Pacific J. Math. 207 (2002), no. 2, 345-358. MR.1972249 (2004c:52015)

[9] M. Götz, On the distribution of weighted extremal points on a surface in $\mathbb{R}^{d}, d \geq 3$, Potential Anal. 13 (2000), no. 4, 345-359. MR1804177 (2002a:31006)

[10] P.J. Grabner, Erdös-Turán type discrepancy bounds, Monatsh. Math. 111 (1991), no. 2, 127-135. MR1100852 (92f:11108)

[11] D.P. Hardin and E.B. Saff, Discretizing manifolds via minimum energy points, Notices Am. Math. Soc. 51 (2004), no. 10, 1186-1194. MR2104914 (2006a:41049)

[12] K. Hesse, A lower bound for the worst-case cubature error on spheres of arbitrary dimension, Numer. Math. 103 (2006), no. 3, 413-433. MR2221056 (2007b:41050)

[13] K. Hesse and I.H. Sloan, Optimal lower bounds for cubature error on the sphere $S^{2}$, J. Complexity 21 (2005), no. 6, 790-803. MR2182445 (2006m:65047) 
[14] Worst-case errors in a Sobolev space setting for cubature over the sphere $S^{2}$, Bull. Austral. Math. Soc. 71 (2005), no. 1, 81-105. MR2127668 (2005k:41090)

[15] _ Cubature over the sphere $S^{2}$ in Sobolev spaces of arbitrary order, J. Approx. Theory 141 (2006), no. 2, 118-133. MR2252093

[16] J. Korevaar, Fekete extreme points and related problems, Approximation theory and function series (Budapest, 1995), Bolyai Soc. Math. Stud., vol. 5, János Bolyai Math. Soc., Budapest, 1996, pp. 35-62. MR1432660 (98c:31004)

[17] A.B.J. Kuijlaars and E.B. Saff, Asymptotics for minimal discrete energy on the sphere, Trans. Am. Math. Soc. 350 (1998), no. 2, 523-538. MR.1458327 (98e:11092)

[18] L. Kuipers and H. Niederreiter, Uniform distribution of sequences, Pure and Applied Mathematics, John Wiley \& Sons, a Wiley- Interscience Publication, New York, 1974. MR0419394 $(54: 7415)$

[19] N.S. Landkof, Foundations of modern potential theory, Springer-Verlag, New York, 1972, Translated from the Russian by A. P. Doohovskoy, Die Grundlehren der mathematischen Wissenschaften, Band 180. MR0350027 (50:2520)

[20] P. Leopardi, A partition of the unit sphere into regions of equal area and small diameter, Electron. Trans. Numer. Anal. 25 (2006), 309-327 (electronic). MR2280380

[21] X.-J. Li and J.D. Vaaler, Some trigonometric extremal functions and Erdös-Turàn type inequalities, Indiana Univ. Math. J. 48 (1999), no. 1, 183-236. MR1722198(2001a:11136)

[22] C. Müller, Spherical Harmonics, Lecture Notes in Mathematics, vol. 17, Springer-Verlag, 1966.

[23] R.B. Paris and D. Kaminski, Asymptotics and Mellin-Barnes integrals, Encyclopedia of Mathematics and its Applications, vol. 85, Cambridge University Press, Cambridge, 2001. MR1854469 (2002h:33001)

[24] A.P. Prudnikov, Yu.A. Brychkov, and O.I. Marichev, Integrals and series, vol. 1, Gordon \& Breach Science Publishers, New York, 1986. MR874986 (88f:00013)

[25] E.D. Rainville, Special functions, Chelsea Publishing Co., Bronx, New York, 1971. MR.0393590(52:14399)

[26] E.A. Rakhmanov, E.B. Saff, and Y.M. Zhou, Minimal discrete energy on the sphere, Math. Res. Lett. 1 (1994), no. 6, 647-662. MR1306011(96e:78011)

[27] E.B. Saff and A.B.J. Kuijlaars, Distributing many points on a sphere, Math. Intell. 19 (1997), no. 1, 5-11. MR.1439152 (98h:70011)

[28] P. Sjögren, Estimates of mass distributions from their potentials and energies, Ark. Mat. 10 (1972), 59-77. MR0310268 (46:9369)

[29] S. Smale, Mathematical problems for the next century, Math. Intelligencer 20 (1998), no. 2, 7-15. MR1631413 (99h:01033)

[30] W.J.H. Stortelder, J.J.B. de Swart, and J.D. Pintér, Finding elliptic Fekete points sets: two numerical solution approaches, J. Comput. Appl. Math. 130 (2001), no. 1-2, 205-216. MR1827981 (2002b:90137)

[31] G. Wagner, On the product of distances to a point set on a sphere, J. Aust. Math. Soc., Ser. A 47 (1989), no. 3, 466-482. MR.1018975 (90j:11080)

[32] _ On means of distances on the surface of a sphere. II: Upper bounds, Pac. J. Math. 154 (1992), no. 2, 381-396. MR.1159518 (93b:52007)

Center for Constructive Approximation, Department of Mathematics, Vanderbilt University, NAshVille, Tennessee 37240

E-mail address: Johann.Brauchart@Vanderbilt.Edu 